\title{
HYBASE: HYperspectral BAnd SElection
}

\author{
Piet B.W. Schwering, Herman H.P.Th. Bekman and Harm H. van Seijen \\ TNO Defence, Security and Safety, P.O. Box 96864, NL-2509 JG The Hague, Netherlands, piet.schwering@tno.nl
}

\begin{abstract}
Band selection is essential in the design of multispectral sensor systems. This paper describes the TNO hyperspectral band selection tool HYBASE. It calculates the optimum band positions given the number of bands and the width of the spectral bands. HYBASE is used to assess the minimum number of spectral bands that is required to get the best target background contrast. The band selection algorithm is described along with a description of the graphical user interface. HYBASE is tested on a representative dataset. The test results shed new light on the optimum band selection. HYBASE is developed for the Royal Netherlands Army to in vestigate the benefit brought by hyper- or multispectral sensors in comparison to present day broad band sensors. HYBASE is tested in European field trials.
\end{abstract}

Keywords: Hyperspectral Imaging, Multispectral, Band selection, Infrared, Detection, Recognition, Identification.

\section{INTRODUCTION}

The ever growing complexity of the modern battlefield makes it more difficult to generate a recognized environmental picture. Constraints like less availability of qualified personnel and changing battleground conditions contribute to this growth. This resulted in a need for sensors which are able to discriminate threats better than sensors that are built today and $\mathrm{s}$ ensors $\mathrm{t}$ hat can be adapted to a $\mathrm{n}$ ew operation al $\mathrm{s}$ cenario. $\mathrm{T}$ here a re s everal e merging techn ologies for discriminative imaging; one of them is multi- and hyperspectral sensors.

Present day hyperspectral sensors may contain a high number of spectral bands ranging typically from 100 to 200 . The penalty for using such a high number of spectral ban ds is that the signal to noise rat io decreas es. Als o operation al systems will be co mplex a nd ex pensive beca use of the wavelength dis crimination e lement ins ide the hy perspectral sensor, this is es pecially true for the infrared wavelength range. If the complete hyperspectral i mage cube has to be processed for the detection of targets making use of both spectral and spatial target characteristics the huge amount of data of a hyperspectral image cube is troublesome. This complicates a near real time image processing solution. B and selection is therefore seen as an important step in realizing operational hyper/multi spectral imaging solutions

Most research involving band selection has been focusing only on the location of the bands. However, for a multispectal configuration very narrow ba nds are not practical, beca use this would requ ire long integration times to get a good signal-to-noise ratio. O ur research therefore n ot o nly looks at the location of $t$ he ban ds but al so at the width of the bands. In previous research a first at tempt has bee $n$ made by dev eloping a $n$ algorithm that first determines the best locations for the bands and than the best width of the bands. This however does not allow for a co mparison bet ween broad and narrow bands. Therefore a n ew algorithm has been written that is capable of finding a specified number of bands of a s pecified width. T wo version of this al gorithm $h$ ave been dev eloped, a f ast on e that ca $n$ qu ickly find a solution bu $\mathrm{t}$ does not guarantee to find $\mathrm{t}$ he bes $\mathrm{t}$ ban $\mathrm{ds}$ an $\mathrm{d}$ a $\mathrm{n}$ opti mal al gorithm, which searches all pos sible combinations but as a consequence takes a lot longer and can only be used if the number of required bands is small.

\section{BAND SELECTION ALGORITHMS}

In our pre vious res earch (Withagen et al., 2001) a fi rst at tempt has been made by developing an algorithm that first determines the best locations for the bands and than the best width of the bands. This ho wever does not allow for a comparison between broad and $\mathrm{n}$ arrow bands. Therefore a new algorithm has been written that is capable of finding a

Algorithms and Technologies for Multispectral, Hyperspectral, and Ultraspectral Imagery XV,

edited by Sylvia S. Shen, Paul E. Lewis, Proc. of SPIE Vol. 7334, 733422 · @ 2009 SPIE

CCC code: 0277-786X/09/\$18 - doi: 10.1117/12.818468

Proc. of SPIE Vol. 7334 733422-1 
specified number of bands of a specified width. Two versions of this algorithm have been developed, a fast one that can quickly find a so lution but d oes no $t$ gua rantee to fi nd the b est $b$ ands a nd a $n$ o ptimal a lgorithm, which se arches a ll possible combinations but as a consequence takes a lot longer and can only be used if the number of required bands is small.

To ev aluate a ba nd co mbination the band selection al gorithm uses a dis tance measure that quantifies the separation between classes. We used two different distance measures (Landgrebe, 2003). The Mahalanobis distance is defined as:

$$
D=\sqrt{\left[\mu_{1}-\mu_{2}\right] \Sigma^{-1}\left[\mu_{1}-\mu_{2}\right]^{T}}
$$

Where $\mu_{1}$ and $\mu_{2}$ are the clas s averages of clas $s$ (targ et) and 2 (back ground) and $\Sigma$ is the cov ariance matrix of the classes.

When using the Mahalanobis distance measure one has to keep in mind that the following assumptions are made:

- The distributions of the classes are multivariate Gaussian distributions.

- The covariance matrix of these distributions is the same for all classes.

- The total number of pixels is large enough to accurately describe the covariance matrix (a rule of thumb is that the number of pixels should be at least 10 times the number of dimensions).

This dis tance measure is $\mathrm{i}$ mplemented $\mathrm{b}$ y first trans forming $\mathrm{t}$ he $\mathrm{f}$ eature-space an $\mathrm{d}$ then calcu lating th e $\mathrm{E}$ uclidian distance bet ween the ce ntres of the clas ses in this trans formed feature space. The tran sformation makes us e of the average co variance matrix of th e diff erent cla sses involved. The data is tra nsformed to a diff erent feature-space by multiplying it with the eigenvectors of this covariance matrix. The effect of this transformation is that the data is d ecorrelated. (for color images, please see electronic version of manuscript)
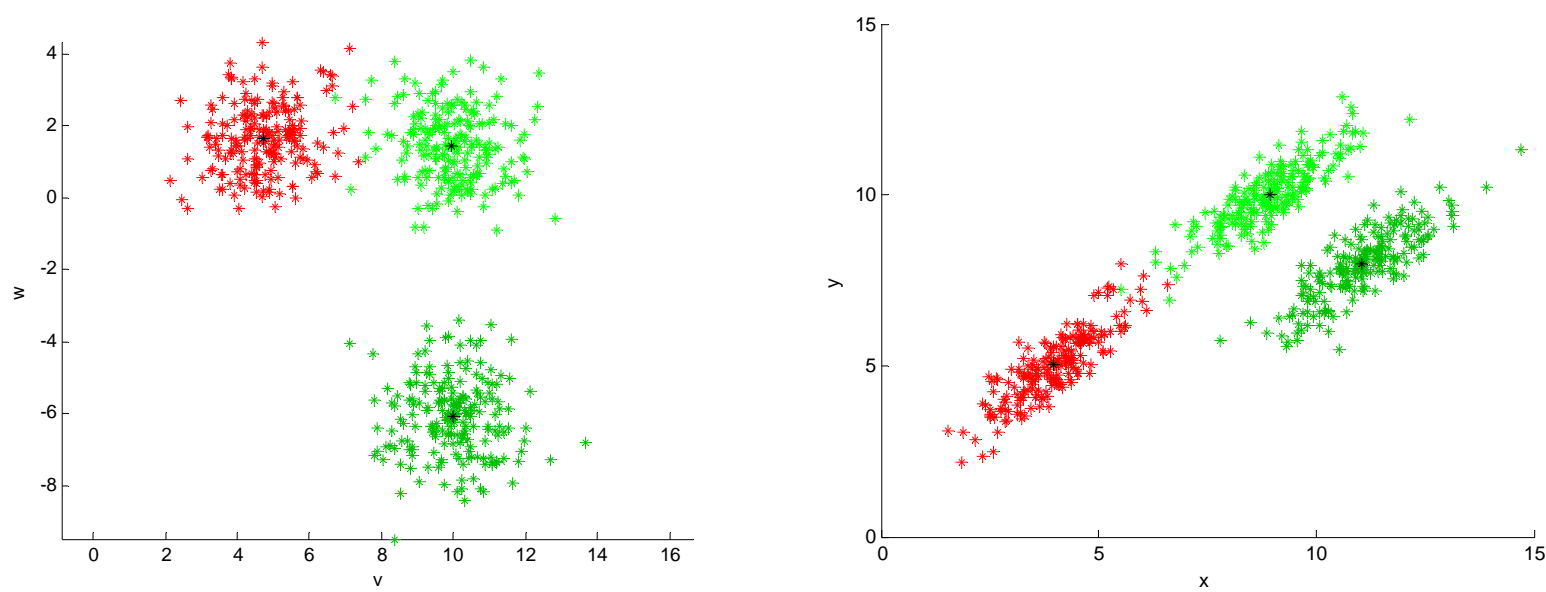

Figure 1: $\quad$ Example of a 2D feature-space (left) and its transformation (right).

The advantage of this can be seen in Figure 1. In the original feature-space (left figure) the distance between BG1 (light green) a nd B G2 (dark green) is lar ger than the dis tance bet ween B G1 (li ght green) and T 1 (red), an d h ence the separability between BG1 and BG2 is better. In the transformed feature-space (right figure) the classes which are most easy to separate also have the highest (Euclidian) distance. In the transformed feature-space the (Euclidian) distance is calculated between the centres of the different classes. The resulting set of distances is stored in a distance matrix. From this matrix a final single distance value can be derived in several ways depending on the experimental requirements (for example the minimum value of this matrix can be tak en). B ecause we want to dis tinguish bet ween backg round and 
target classes we choose the smallest distance between a target and a background class. This final distance value we will refer to as the quality (of a band combination). In the band selection algorithm this quality is maximized.

The Bhattacharyya distance is another distance measure that measures the distance between two multivariate Gaussian distributions. It is defined as:

$$
B=\frac{1}{8}\left[\mu_{1}-\mu_{2}\right]\left[\frac{\Sigma_{1}+\Sigma_{2}}{2}\right]^{-1}\left[\mu_{1}-\mu_{2}\right]^{T}+\frac{1}{2} \ln \frac{\mid 1 / 2\left[\Sigma_{1}+\Sigma_{2}||\right.}{\sqrt{\left|\Sigma_{1}\right|\left|\Sigma_{2}\right|}}
$$

One important difference with the Mahalanobis distance is that it does not take the a verage covariance matrix of the classes but keeps the clas s covariance matrices. The price th at has to be paid is that now each clas $\mathrm{s}$ as to con tain enough pixels to describe its covariance matrix accurately.

\section{OPTIMIZATION OF BAND SELECTION}

To $f$ ind the bes $t$ ban d com bination t wo alg orithms h ave been dev eloped. On e alg orithm searches all pos sible ban $d$ combinations. This algorithm can only be used if the number of required bands is small $(<4)$ because calculation times increase exponentially with the number of bands. Therefore another algorithm has been written that searches in a more time efficient way, but as a consequence it is not guaranteed to find the best band combination.

The al gorithms are i mplemented in Mat lab® and make us e of the toolbox PR Tools, a toolbox of fered for free for academic res earch by the Univ ersity of Del ft in The Neth erlands (reference 3). $\mathrm{T}$ he $\mathrm{m}$ ain data-object of PRTools is called the dataset. In this dataset a large number of objects can be stored, each object consisting of a certain amount of features. We use this dataset-type to store our pixel-data. The dataset-object also makes it possible to label each object with an integer value, which can be used to divide the pixels in different classes.

We have analyzed the effects of two algorithms:

- Algorithm 1 is the fast alg orithm. The way it select $\mathrm{s}$ its $\mathrm{b}$ ands is $\mathrm{b}$ y first selecting the $\mathrm{b}$ and with the highest quality. Then it searches for a second band that in combination with the already found band gives the highest quality. Then it sear ches for $a$ th ird $b$ and in the sa me way and so on till th e number of required $b$ ands are found.

- Algorithm 2 (called the optimum al gorithm) searches every possible combination of bands, which guarantees that it will find the ban $\mathrm{d}$ co mbination with $\mathrm{t}$ he highest qu ality. $\mathrm{B}$ ecause ca lculation $\mathrm{t}$ imes i ncrease exponentially with the number of bands, it can on ly be used if the number of bands required is small. The algorithm can al so be used in a s ub-optimal way, by defining a s tep-parameter (see below) higher than 1, in which case the algorithm gets faster. Besides a poten tially better solution, alg orithm 2 has another advantage with respect to algorithm 1: because it calculates the quality for each combination, an overview of all qualities can be made, giving extra insight in the problem.

To do the classification, the QDC (quadratic discriminant classifier) classifier provided by PRTools is used. This is a normal densities based quadratic classifier. For the algorithms several inputs are needed: number of background classes, bandwidth, s hape, dis tance t ype, ov erlap, qu ality criteria. For alg orithm 2 we als o s et a s tep an d ti me es timation parameter. For TimeEstimation, if 1, the algorithm makes an estimate of the calculation time by calculating how many combinations it will have to evaluate and multiplying this with the quality evaluation-time, which it $\mathrm{g}$ ets by making 5 evaluations and taking the average. A Step parameter (default is 1 ) is d efined to use the alg orithm in a f aster, suboptimal way. The idea beh ind th is para meter is that when for ex ample a ban dwidth of $30 \mathrm{f}$ eatures is used, the band consisting of features 1 through 30 will almost be exactly the same as band 2-31. By setting a step of for example 3, the algorithm will only take into account bands 1-30, 4-33, 7-36 and so on, which can greatly decrease the calculation-time without sacrificing much of the optimality of the solution. 
Table 1: $\quad$ Calculation times for algorithm 1, with max_overlap $=0$ and band_width $=1$. The number of pixels used is 1000 .

\begin{tabular}{|l|l|l|}
\hline number of bands & $\begin{array}{l}\text { calculation time } \\
\text { Bhattacharyya [s] }\end{array}$ & $\begin{array}{l}\text { calculation time } \\
\text { Mahalanobis [s] }\end{array}$ \\
\hline $\mathbf{2}$ & 8.516 .0 & \\
\hline $\mathbf{4}$ & 19.031 .9 & \\
\hline $\mathbf{6}$ & 30.947 .7 & \\
\hline $\mathbf{8}$ & 43.563 .7 & \\
\hline $\mathbf{1 0}$ & 57.380 .3 & \\
\hline
\end{tabular}

If the overlap parameter is set to 0 (no overlap allowed), the bandwidth has also some influence on the calculation time. The larger the bandwidth the faster the algorithm will be becau se after the first band has been picked all features that make up this band are ex cluded for the following bands so effectively the total number of features decreases. Table 1 shows the calc ulation ti mes of the Mat lab i mplementation of al gorithm 1 for sev eral $n$ umbers of $b$ ands using the Bhattacharyya or the Mahalanobis distance. The bandwidth used is 1 . T he number of pixels used is 1000 . F rom this table can be concluded that the calculation of the Bhattacharyya distance takes on average about $30 \%$ less time then the calculation of the Mahalanobis distance.

Calculation times for algorithm 2 are a lot higher than those of algorithm 1 . The relation between the calculation time, the number of bands and the total number of features is:

$$
\text { calc_time } \sim \text { no_pixels } \cdot \frac{\text { total_features! }}{\text { no_bands!(total_features-no_bands)! }}
$$

Depending on the value of the max_overlap parameter, the bandwidth also has a big influence on the calculation time. In $\mathrm{t}$ able $2 \mathrm{~s}$ ome cal culation $\mathrm{t}$ imes are given for $\mathrm{t}$ hree di fferent ban dwidths a nd 2 di fferent num bers of ban ds. max_overlap is set at 0 and the distance measure is Bhattacharyya. The Mahalanobis distance measure shows the same pattern but the times are about $30 \%$ higher.

Table 2: $\quad$ Calculation times (hh:mm:ss) for algorithm 2, using the Bhattacharyya distance measure, with max_overlap $=0$ and band_width $=1,10$ and 30. The number of pixels used is 1000. For step parameter $=1,2$.

\begin{tabular}{|c|c|c|c|c|c|c|}
\hline \multirow{2}{*}{$\begin{array}{l}\text { number of } \\
\text { bands }\end{array}$} & \multicolumn{3}{|l|}{ Step: 1} & \multicolumn{3}{|l|}{ Step: 2} \\
\hline & 1 & 10 & 30 & 1 & 10 & 30 \\
\hline 2 & $00: 10: 03$ & $00: 07: 20$ & 00:04:58 00: & $02: 1000:$ & 01:50 00: & $00: 54$ \\
\hline 3 & $10: 33: 20$ & 07:30:00 & 02:08:20 01: & 13:20 00: & 48:57 00: & $16: 10$ \\
\hline
\end{tabular}

\section{GRAPHICAL USER INTERFACE (GUI)}

The number of required spectral bands is assessed with HYBASE in a number of steps in a Matlab® environment. The first requirement is that the user has to i nput a hyperspectral image cube in which target and background are pres ent. Subsequently regions in the image are selected and attributed to either background or target. Target boxes are coloured in red, background boxes in white. When all relevant target and background areas are selected the spectra of all pi xels inside either the target or the background boxes are plotted at the bottom panel of the GUI (see Figure 2). 


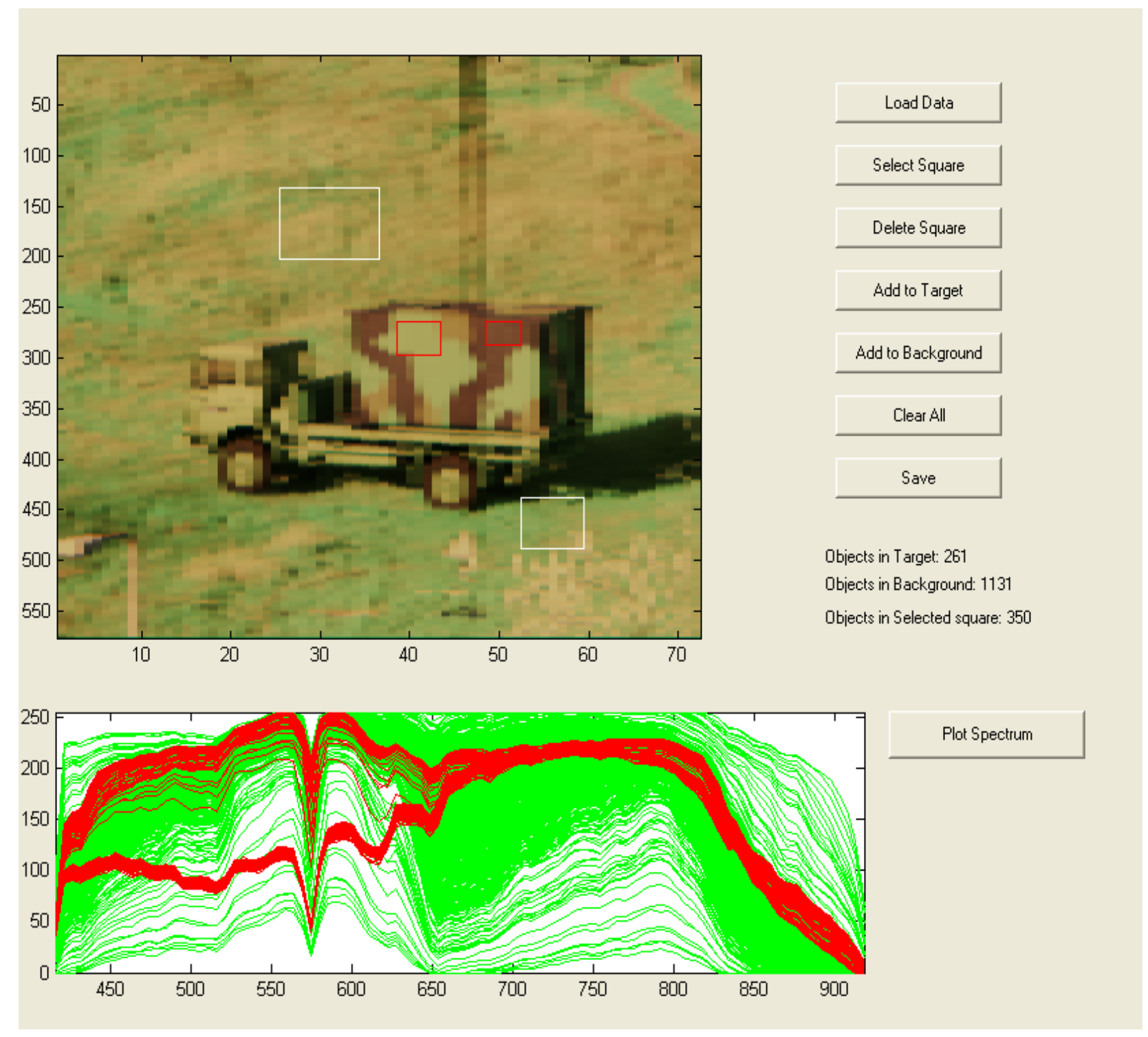

\section{Figure 2: $\quad$ Spectra of target areas and background areas are selected via Matlab® GUI}

(for color images, please see electronic version of manuscript)

Within the feature (or spectral band) selection tool the feature width and the maximum allowed overlap are input. Now the optimum position of these features is calculated. The result of the optimum band positions is plotted in the top panel of the GUI (see Figure 3), by vertical lines that are drawn over the spectra. Each band starts with a blue vertical line and ends with a black vertical line. The optimum spectral band positions are also outputted to the Matlab® command line. 


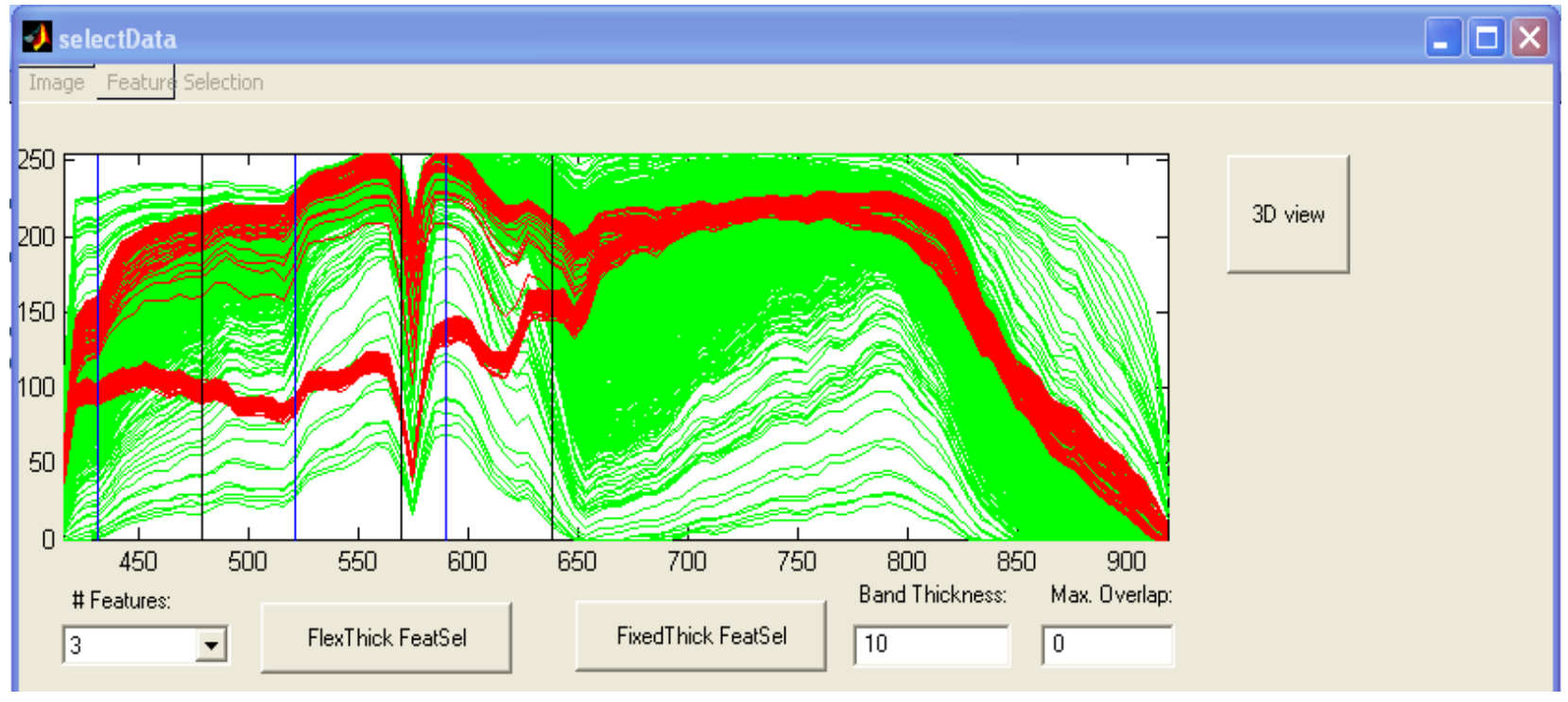

Figure 3: $\quad$ Optimum position of spectral bands are indicated by vertical lines. Blue lines mark start of new band, black lines mark end of band. (for color image, please see electronic version of manuscript)

If exactly three features are selected the position of the pixels in 3-dimensional feature space are plotted (see Figure 4). The $u$ ser can rotate th e cu be $f$ or better $\mathrm{v}$ isualization of $\mathrm{t}$ he $\mathrm{s}$ eparation $\mathrm{t}$ hat $\mathrm{h}$ as been achieved bet ween targ et a nd background pixels using the selected number of features.

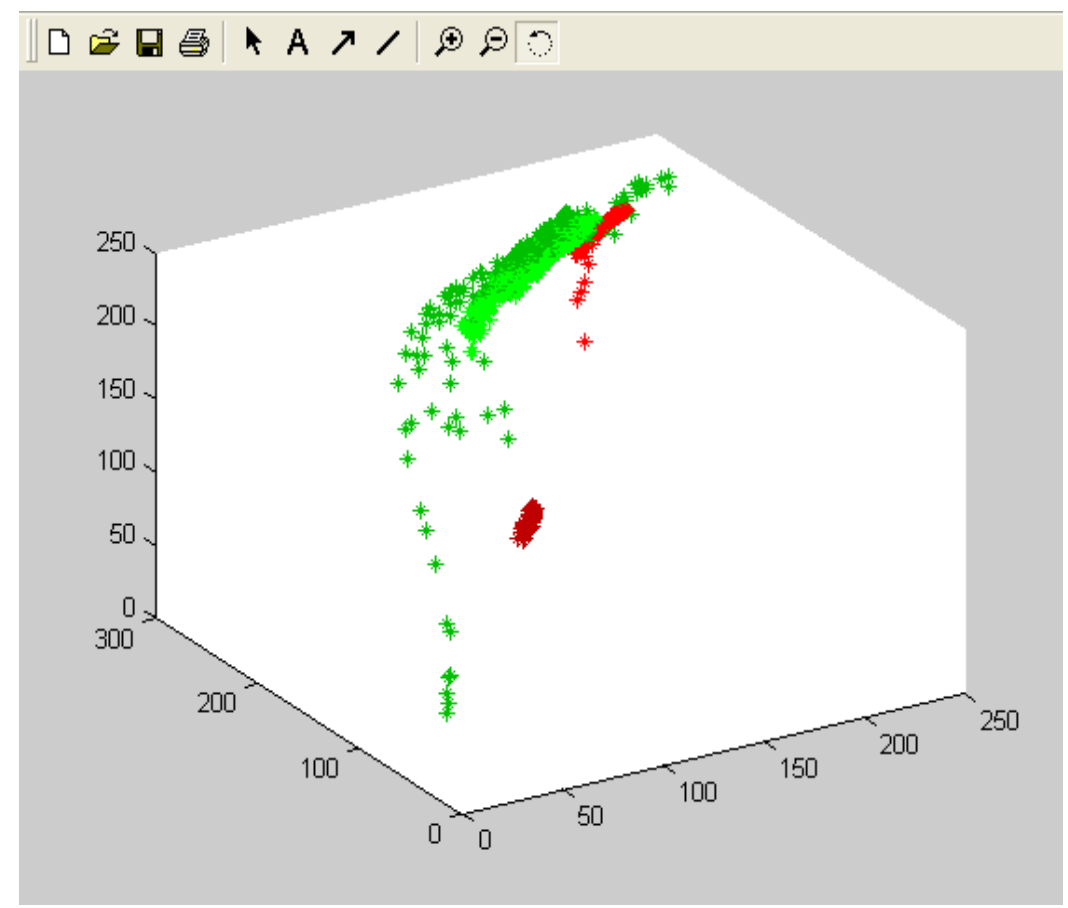

Figure 4: $\quad$ Projection of pixels in 3-dimensional space spanned by three selected features (=spectral bands) 


\section{BAND SELECTION RESULTS}

To see how algorithm 1 (fast) performs compared to the algorithm 2 (slow, but optimal) a co mparison has been made for a representative data set for the case that several targets are used with the following input settings:

bandwidth $=30$

number of bands $=3$

step $=2$

overlap $=0$

The comparison has been made for the Bhattacharyya as well as the Mahalanobis distance. Table 3 summarizes the result.

Table 3: $\quad$ Comparison between algorithm 1 and algorithm 2.

\begin{tabular}{|l|l|l|l|c|}
\hline & $\begin{array}{l}\text { algorithm 1 } \\
\text { bhattacharyya }\end{array}$ & $\begin{array}{l}\text { algorithm 2 } \\
\text { bhattacharyya }\end{array}$ & $\begin{array}{l}\text { algorithm 1 } \\
\text { mahalonobis }\end{array}$ & $\begin{array}{l}\text { algorithm 2 } \\
\text { mahalonobis }\end{array}$ \\
\hline Quality & 0.4745 & 0.5450 & 2.3396 & 2.7131 \\
\hline $\begin{array}{l}\text { QDC class. error } \\
\text { (misclassified pixels) }\end{array}$ & 75 & 76 & 81 & 78 \\
\hline band 1 (feature numbers) & $80-109$ & $81-110$ & $6-35$ & $7-36$ \\
\hline band 2 (feature numbers) & $114-143$ & $117-146$ & $110-139$ & $95-124$ \\
\hline band 3 (feature numbers) & $168-197$ & $169-198$ & $169-198$ & $169-198$ \\
\hline
\end{tabular}

Algorithm 1 performs quite well in finding the maximum quality. For the Bhattacharyya distance, algorithm 1 is $13 \%$ off, and for the Mahalanobis distance it is $14 \%$ off. The difference in classification error is even smaller.

The most surprising thing is that some of the found bands are really different, comparing band 1 for the Bhattacharyya distance and the Ma halanobis dis tance of al gorithm 2 shows very di fferent ban ds, while the classification error i s similar. This raises the question if there are more band combinations that give similar results.

To investigate this, the quality of all band combinations (34220 in total) has been plotted for the Bhattacharyya distance and the Mahalanobis distance. These plots offer a revealing view on the significance of 'best bands'. There are in fact a lot of dif ferent ban d co mbinations that $h$ ave a qu ality cl ose to th e maximum value, es pecially in the cas e of the Bhattacharyya di stance. T he periodic $n$ ature of $t$ he $\mathrm{f}$ igures aris es from $\mathrm{t}$ he $\mathrm{s}$ ystematic way $\mathrm{i} n$ which $\mathrm{t}$ he ban $\mathrm{d}$ combinations were chosen. Because of that a certain band reoccurs every so often.

Having in mind that there is no direct translation of the distance measure into the classification result, it makes sense to not only look at the band combination with the highest distance, but also at the ones that come close to that. If the bands are plotted that are within $10 \%$ of the maximum value for the Bhattacharyya distance., the band around $11 \mu \mathrm{m}$ has the highest contribution to the quality, since it is always present, see figure 5 . W hen this band is chosen in combination with a band between 10 and $10.5 \mu \mathrm{m}$, the choice of the third band doesn't matter anymore. It can be anywhere between 8 and $9.7 \mu \mathrm{m}$. So the contribution of this third band is minimal and in this case just taking the first two bands would probably give a similar classification result.

In figure 5 (right side) also the pixel classification results are plotted as well. The red line in those graphs represents the classification error of the band combination with the highest quality. Its classification result is average compared to the classification when the other band combinations with quality within $10 \%$ of the maximum is being used.

Classification results have also been compared by using the Mahalanobis distance. This time there are only a total of 26 band com binations th at are $\mathrm{w}$ ithin $10 \%$ of the maximum an $\mathrm{dt}$ he ban ds are all arou nd the $\mathrm{s}$ ame wavelengths. Surprisingly, these bands d o n ot s how up in the set of best b ands found using the B hattacharyya criterion. S till, the 
bands found with the Mahalanobis criterion give a co mparable classification result. Apparently, the boundary of $10 \%$ within the maximum could be set lower to include even more band combinations.
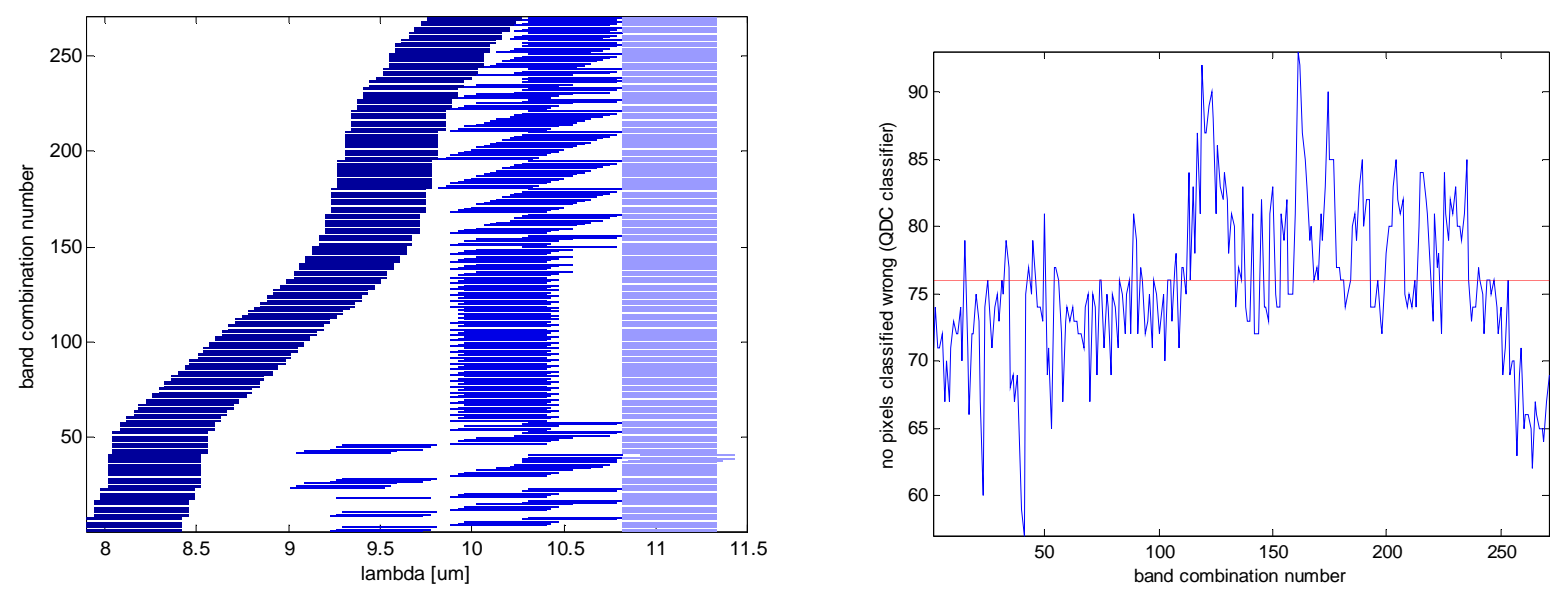

Figure 5: $\quad$ Bands that have a quality (using the Bhattacharyya distance) within 10\% of the maximum quality and associated miss-classifications

\section{APPLICATION OF BAND SELECTION}

HYBASE is typically used in a system design study and these outputs can feed operational studies. Figure 6 shows the location of HYB ASE in this design chain. B ased on a hy perspectral data s et in a relev ant scenario one can make an analysis with $\mathrm{H}$ YBASE of $\mathrm{t}$ he minimum number o $\mathrm{r}$ equired $\mathrm{sp}$ ectral $\mathrm{b}$ ands, their widths a nd $\mathrm{p}$ ositions for th $\mathrm{e}$ targets/backgrounds studied.

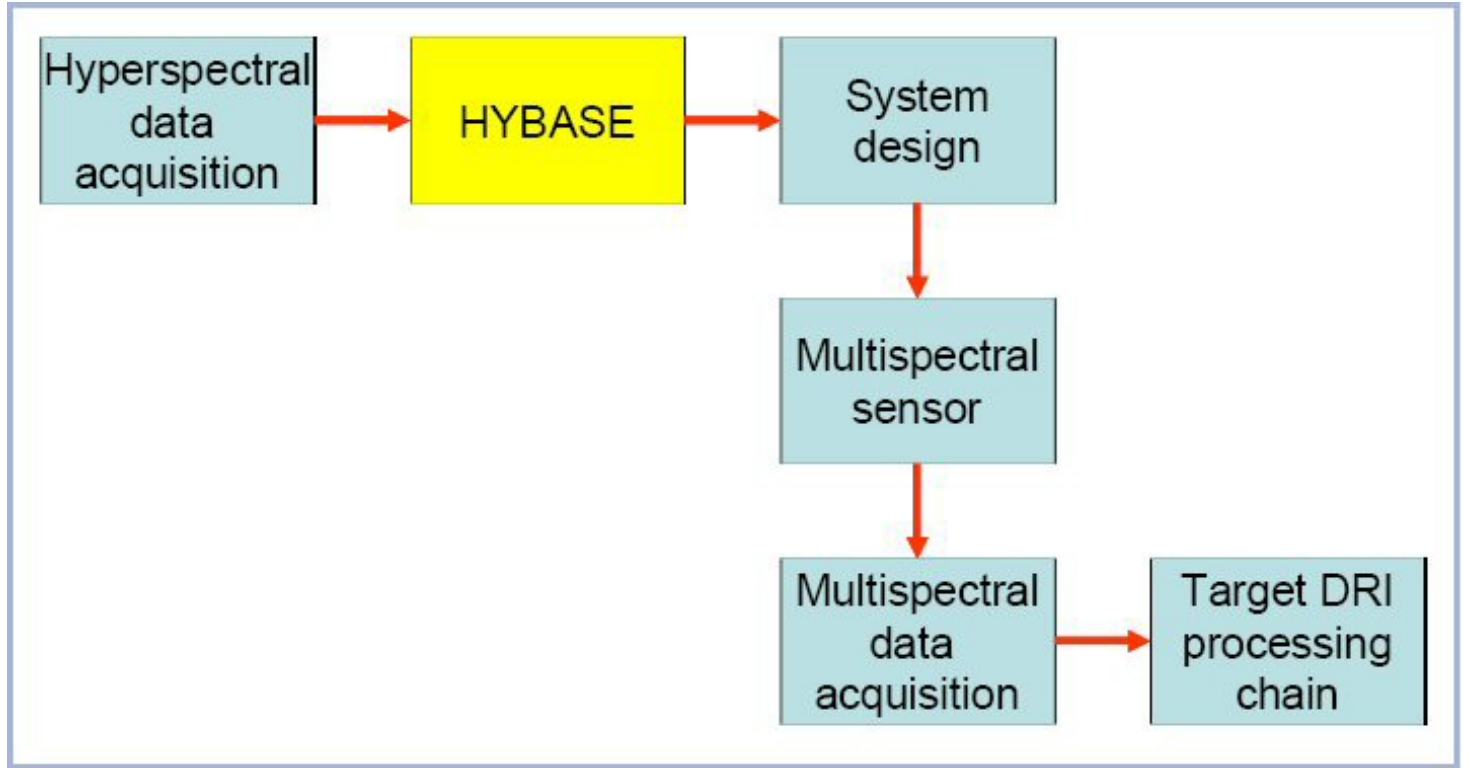

Figure 6: $\quad$ Typical usage of HYBASE in system design 
When a multispectral sensor has been designed and realized the system will be used for data acquisitions. Multispectral target detection/classification tools will then be used to extract the relevant data. The target DRI (detection, recognition, identificaton) proces sing $\mathrm{c}$ hain $\mathrm{f}$ irst pre- processes $\mathrm{t}$ he acqu ired $\mathrm{h}$ ypercubes ( geo $\mathrm{r}$ eferencing, no ise $\mathrm{r}$ eduction, $\mathrm{d}$ ata normalization, temperature emissivity separation). Then targets are bein g detected us ing anomaly and signature based detection method in co mbination with $\mathrm{c}$ hange $\mathrm{d}$ etection. $\mathrm{Sp}$ atial in formation is used to $\mathrm{r}$ educe $\mathrm{f}$ alse alar $\mathrm{m} \mathrm{r}$ ates. Additional se nsor $d$ ata f rom e.g. high r esolution i magers, radar an d/or $3 \mathrm{D}$ laser $\mathrm{r}$ adar is b eing u sed to class ify a nd identify targets in a deci sion f usion proces s. Many of these alg orithms run n ear real-ti me. Potential ap plications of sensor combinations are described in Schwering et al. (2007).

\section{DISCUSSIONS AND CONCLUSIONS}

An effective approach to optimum band selection in hyperspectral imaging has been demonstrated.

Target detection/recognition/identification is part of a more extensive software processing chain as depicted in Figure 6. First $t$ he acq uired hy percubes are bei ng pre -processed ( $\mathrm{g}$ eo $\mathrm{r}$ eferencing, noise $\mathrm{r}$ eduction, $\mathrm{d}$ ata normalization, temperature e missivity separation). $\mathrm{T}$ hen targets are bei ng det ected using a nomaly a nd s ignature bas ed det ection method in combination with change detection. Spatial information is used to reduce false alarm rates. Additional sensor data from e.g. high resolution imagers, radar an d/or 3D laser radar i s being used to classify and identify targets in a decision fusion process. TNO has access to many of the tools in the processing chain which TNO can offer in addition to the HYBASE band selection tool.

Below are listed the conclusions from our research. What has to be kept in mind is that the conclusions are based upon one dataset with a frequency range from $7.7 \mu \mathrm{m}$ to $12.1 \mu \mathrm{m}$, so mainly emissivity is measured:

- Algorithm 1 ( $\mathrm{t}$ he fast al gorithm) perf orms g ood co mpared t $\mathrm{o}$ al gorithm 2 ( $\mathrm{t}$ he opt imal al gorithm). $\mathrm{T}$ he ba nd combinations found by algorithm 1 have a quality value within $15 \%$ of the quality found by algorithm 2 , while the calculation time is a lot smaller.

- Using the Bhattacharyya distance as a measure for the separation of the different classes gives comparable results as the Mahalanobis distance.

- Although no thorough study has been done between the relation of the quality and the classification error, in some cases the difference in classification error can be very big for similar qualities (up to $100 \%$ difference)

- Often, there is a whole set of different band combinations that have a comparable quality and classification result. This $\mathrm{s}$ et i s re vealed $\mathrm{b}$ y plott ing $\mathrm{t}$ he ban $\mathrm{d}$ co mbinations having a $\mathrm{q}$ uality within a $\mathrm{c}$ ertain percen tage $\mathrm{o} f \mathrm{t}$ he maximum quality.

- As a consequence of the above two points, the band combination with the highest quality does not necessarily have the lowest classification error.

- The location of the best bands depends strongly on the choice of target and backgrounds.

- For a good classification result clean spectra of the targets are required. Target masks for semi-hidden targets are useless, since they contain target as well as background pixels.

- If the number of ba nds i ncreases the qu ality i ncreases a nd th e cla ssification error de creases. Although ot her research shows that there is an optimal number of bands for the classification error, th is did n ot show up in our results. This opti mum is $d$ ue to th e fact, th at when the number of ba nds i ncreases, statistical values used to describe the feature-space like the covariance matrix can be predicted less accurate. That this optimum did not turn up in our results is probably due to the fact that we used the areas that were classified also to train the classifier.

The influence of the bandwidth on the quality is substantially less than the influence of the number of bands. This is probably becaus e the s pectra in the thermal infrared reg ion $(7.7 \mu \mathrm{m}$ to $12.1 \mu \mathrm{m})$ i nvolved did $\mathrm{n}$ ot have a ny sharp features. There is also no clear relation between the bandwidth and the quality, sometimes the quality increases. 


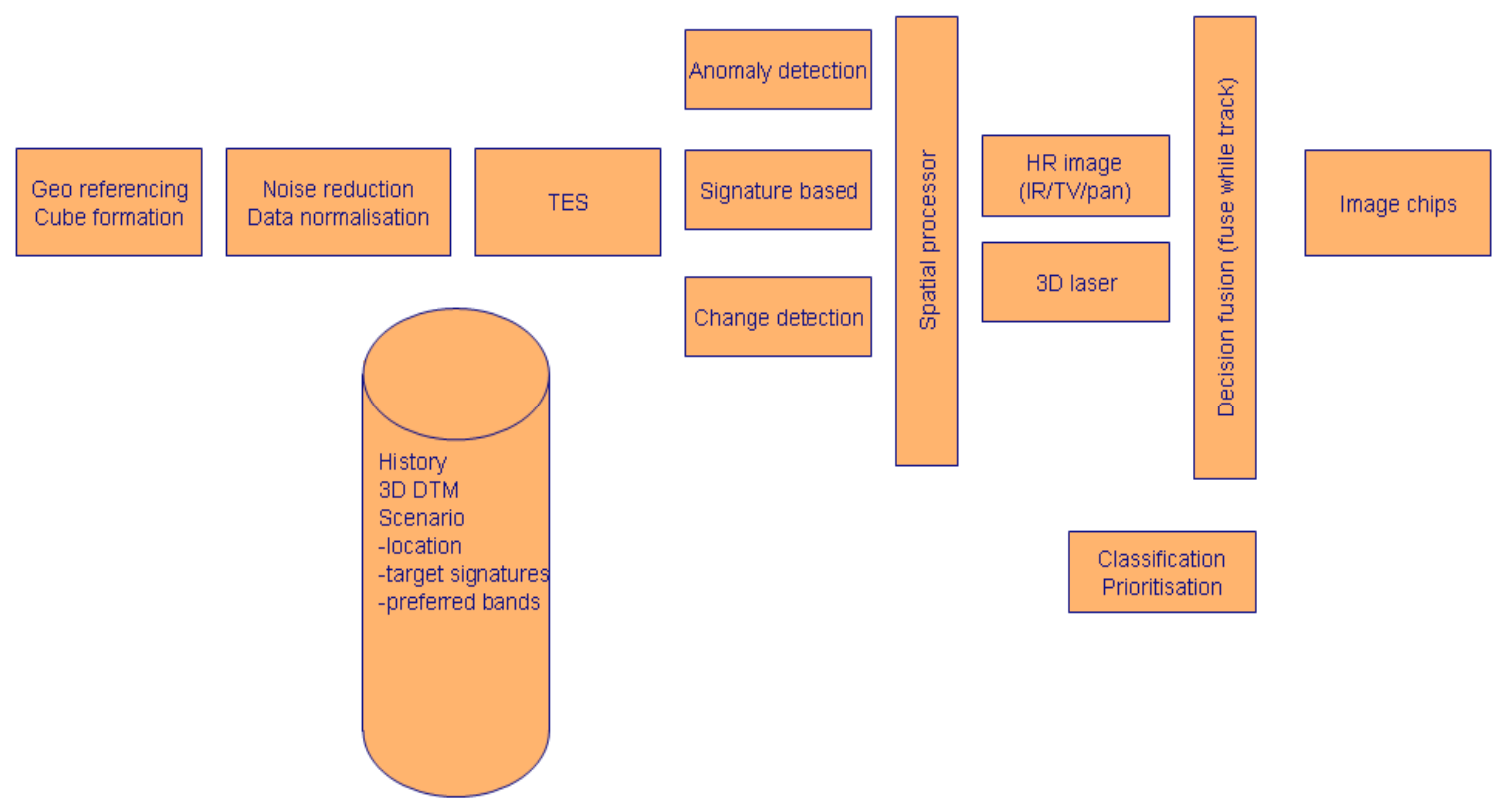

Figure 7: $\quad$ Multi spectral image processing chain

If the complete hyperspectral image cube has to be proces sed for the detection of targets making use of both spectral and s patial tar get $\mathrm{c}$ haracteristics $\mathrm{t}$ he huge a mount of $\mathrm{d}$ ata of a hyperspectral $\mathrm{i}$ mage cu be is tro ublesome. $\mathrm{T}$ his complicates a near real ti me i mage proces sing s olution. Band s election is therefore an i mportant $\mathrm{s}$ tep in real izing operational h yper/multi sp ectral i maging so lutions. I $n$ Fi gure 7 we p resent th e b asic T NO processing ch ain for automatic targ et data proces sing of hyperspectral i mage information. This serves as the big pictu re in the res earch, consisting of real-time on-line steps, combined with supporting off-line data mining activities.

Most research involving band selection has been focusing only on the location of the bands. However, for a multispectal configuration very $n$ arrow ba nds are not practical, becaus e th is would require lar ge integration times to get a good signal-to-noise ratio. $\mathrm{O}$ ur res earch therefore not only looked at the location of the bands but als o at the width of the bands.

\section{ACKNOWLEDGEMENTS}

We g ratefully ac knowledge the stimulating di scussions with ou r col league C .J. den Hollander and financial support from the NL MOD in programme V513.

\section{REFERENCES}

1. P.J. W ithagen, E. den B reejen, et. al. “ Band s election $\mathrm{f}$ rom a hy perspectral data-cu be $\mathrm{f}$ or a real- time multispectral 3CCD camera", Proceedings of SPIE Vol. 4381, pp 84-93, 2001

2. Landgrebe, D.A., "Signal Theory Methods In Multispectral Remote Sensing", Hoboken, NJ: Wiley, 2003

3. University of Delft, www.prtools.org

4. Schwering P .B.W., va $\mathrm{n}$ d en B roek S.P ., va $\mathrm{n}$ I ersel M ., 200 7, i n ' Infrared T echnology a nd A pplications XXXIII', eds. B.F. Andresen, SPIE Vol. 6542-100, 654230, Orl ando Florida (US A), April 9- 13, 2007: "EO System Concepts in the Littoral" 
5. Briottet X, Boucher, Y., Dimmeler, A., Malaplate, A., Cini, A., Diani, M., Bekman, H., Schwering, P., Skauli, T., Kas en, I., R enhorn, I., Klas en, L., Gil more, M., Ox ford, D.: 2006, in ' Targets an d Back grounds: XII, Characterization an d R epresentation', ed. W .R. W atkins, D . Cl ement, SP IE V ol. 6 239-paper163, O rlando Florida (USA), April 17-18 2006: "Military Applications of Hyperspectral Imagery" 